\title{
More than Half the Days
}

National Cancer Institute

\section{Source}

National Cancer Institute. More than Half the Days. NCI Thesaurus. Code C107223.

A time period that includes one-half of the total number of days plus at least one more. 